\title{
Global aerial flyways allow efficient travelling
}

\author{
B. Kranstauber, ${ }^{1,2 *}$ R. Weinzierl, ${ }^{1,2}$ \\ M. Wikelski, ${ }^{1,2}$ and K. Safi, ${ }^{1,2}$ \\ ${ }^{1}$ Department for Migration and \\ Immuno Ecology Max Plank Insti \\ tute for Ornithology 78315 Radolf \\ zell, Germany \\ ${ }^{2}$ Department of Biology, University \\ of Konstanz 78464, Konstanz \\ Germany
}

*Correspondence: $E$ mail: kranstau ber@orn.mpg.de

\begin{abstract}
Birds migrate over vast distances at substantial costs. The highly dynamic nature of the air makes the selection of the best travel route difficult. We investigated to what extent migratory birds may optimise migratory route choice with respect to wind, and if route choice can be subject to natural selection. Following the optimal route, calculated using 21 years of empirical global wind data, reduced median travel time by $26.5 \%$ compared to the spatially shortest route. When we used a time-dependent survival model to quantify the adaptive benefit of choosing a fixed wind-optimised route, $84.8 \%$ of pairs of locations yielded a route with a higher survival than the shortest route. This suggests that birds, even if incapable of predicting wind individually, could adjust their migratory routes at a population level. As a consequence, this may result in the emergence of low-cost flyways representing a global network of aerial migratory pathways.
\end{abstract}

\section{Keywords}

Avian migration, evolution of migratory routes, global wind systems, optimal route.

\section{INTRODUCTION}

Some of the most striking avian migration movements involve billions of individuals undertaking journeys stretching over vast distances, often between continents. While travelling from their breeding to their non-breeding grounds, birds not only master the task to accurately navigate between such distant places, but also face the physiological challenge of completing these long-distance movements. Astounding examples for global migrations abound (Alerstam et al. 2003). They overcome obstacles such as the Pacific ocean (Lindström et al. 2011), mountain ranges as high as the Himalayas (Hawkes et al. 2011) and inhospitable deserts (Biebach et al. 2000). Likewise among the longest distances ever reported are the annual travels of the Arctic tern (Sterna paradisaea) weighing only 100 grams yet travelling between the Arctic breeding and the Antarctic non-breeding areas on an annual basis (Egevang et al. 2010). The bar-tailed godwits (Limosa lapponica baueri), for example travel from Alaska to New Zealand and back in a single year requiring large fat reserves (Gill et al. 2009). Minimising the costs of migration is of pivotal importance as extra energy used during migration could result in reduced investment in reproduction or increased mortality. Furthermore, the time spent during migration could be risky due to the unfamiliar environments encountered. This means that migratory strategies are likely to be under strong natural selection (Newton 2006; Sergio et al. 2014).

In many species, migration is thought to run along distinct corridors, characterised by spatial aggregations of a large proportion of the migrating population, which often use specific routes persistently resulting in migratory corridors, or flyways (Berthold 2001; Newton 2007). Sometimes, the advantage of choosing corridors appears obvious. For example in the straight of Gibraltar, in Israel, or central America, the corridors are likely the consequence of birds avoiding crossing extensive water bodies, while minimising the travel distance between their Northern and Southern destinations (Newton
2007). In many cases, migration corridors do not follow the shortest connection between the start and end of the journeys, the great circle route (Alerstam 2011). Other pathways are thought to connect patches of suitable habitat along the journey, these so called stopovers sites allow small birds in particular to balance migration and maintaining the energy reserves needed to migrate. Longer than minimal travel routes may thus provide essential resources required for successful completion of the journey such as feeding opportunities (Lindström et al. 2011) or safe moulting sites (Gehrold et al. 2014). An alternative explanation for such spatial detours is that the birds can reduce their energy expenditure due to atmospheric assistance. For aerial animals, wind is a major factor determining the speed of locomotion and therefore its cost (Sapir et al. 2011). Head and tail wind influence ground speed and thus the energetic expenses directly, whereas side winds cause deviations from a targeted migration pathway making navigational correction mandatory at the cost of ground speed (Wikelski et al. 2003; Safi et al. 2013). Atmospheric conditions should thus govern the global migratory routes observed in aerial organisms (Alerstam 1979; Alerstam et al. 2003; Liechti 2006).

While some species take advantage of social information to learn migratory routes many more species, particularly those that migrate at night migrate without social input. Cuckoos, that fly from Europe to Africa do so on their own spontaneously and without parental influences (Willemoes et al. 2014). Similarly, in many bird species, juveniles and adults show a different timing for the onset of migration, rendering social information from experienced individuals of the same population unlikely. Evidence for innate migratory behaviour is then unsurprising and indicates that natural selection is important in the genetic propensity of migratory programs. Heritable components in the direction and the timing of the onset of nocturnal restlessness are considered to relate to such innate migratory routes (Pulido et al. 2001; Liedvogel et al. 2011).

Although birds should benefit from wind support, it is not clear whether an individual bird can travel between any two 
distant localities more efficiently using the winds. The choice of an optimal route, with respect to the wind support obtained, could result in a substantial reduction of travel time compared to the shortest geographic route. The difference between such wind-supported vs. distance-optimised routes indicates the potential for energetic optimisation of a specific journey at a specific time.

Optimal routes are specific to a starting time because the atmospheric conditions vary over time and as a consequence the optimal route. On the basis of the question of energy optimisation along travel routes, we model the optimal route with respect to wind at four atmospheric levels, based on 21 years of atmospheric data at a $6 \mathrm{~h}$ interval between any two locations in the world. We assume that (1) a bird using flapping flight, (2) minimises the total duration of a given journey (Hedenström 2008) and (3) travels over a hexagonal grid (Fig. 1). For the sake of simplicity, we assume the air speed of the bird to be constant at $10 \mathrm{~m} \mathrm{~s}^{1}$, whereas tail and cross winds increase or decrease ground speed linearly and thereby influence travel time. The empirical evidence suggests that birds generally try to maintain ground speed, within some limits, by adjusting their air speed and thus investing more or less effort into flight (Liechti et al. 1994; Shamoun-Baranes et al. 2007). The simplified assumption of linearity in the increase and decrease in ground speed as a function of wind direction and speed, in conjunction with a constant air speed as used in our models, thus represents an approximation of effort while travelling a specific route, rather than realistic travel times. Whether or not birds maintain ground and/or air speed within some limits, the optimisation of the journey according to our assumptions will capture a mixture of true changes in speed and/or effort, both of which represent important resources. In our model, the birds suspend flight whenever head winds become too strong to make progress in a given direction, but otherwise fly in one long enduring flight which has been shown to be the flight mode for shore birds (e.g. Gill et al. 2009; Klaassen et al. 2011).

An individual bird has to decide, based on local knowledge, and maybe experience, when to start migrating and which route it should take. However, even if wind support results in major time savings, for any given starting time, the optimal route connecting any given pair of start and end locations can only be found by knowing (or forecasting) the future wind conditions for all possible routes. Individual birds are, in contrast to our model, unlikely to be able to know (forecast) wind conditions with enough reliability over the spatial and temporal distances over which they usually migrate.

However, optimal migratory routes could be selected for (or learned and culturally inherited e.g. Mueller et al. 2013) despite these individual inabilities to predict future wind conditions at the time of departure. Provided that wind support is temporally repeatable and energetic and survival benefits may accrue to an individual, specific migratory routes with optimised wind support may emerge under natural selection at a population level. Thus, we predict the emergence of stable migration corridors at the population level, given that wind conditions between locations provide a predictable and repeatable benefit (time and/or energy) over an annual cycle that matches the biological cycle of reproduction and migration.

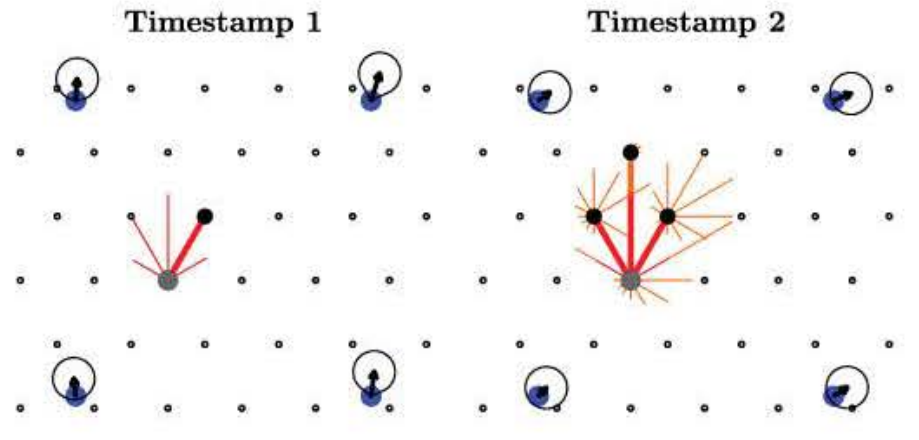

Timestamp 3

Timestamp 4

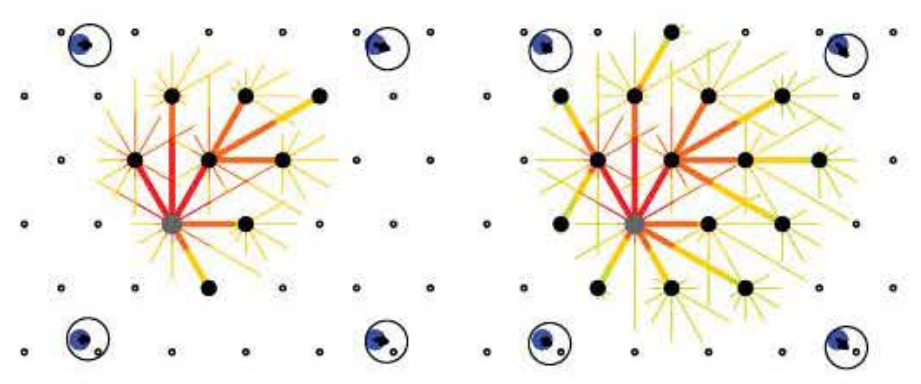

Figure $1 \mathrm{~A}$ visualisation of the algorithm that finds the optimal route. Travel starts at the grey node in all directions without intended destination. Wind is indicated by the arrow at the blue points. The air speed of the bird in each direction is kept constant at $10 \mathrm{~m} \mathrm{~s}^{-1}$. The realised ground speed indicated by the black circle, however, is influenced by wind speed and direction which are interpolated bi linearly to all nodes. When a node has been reached (solid circles), the travel continues from there iterating the calculations by travelling to all surrounding nodes that have not yet been reached. Inclusion of the influence of wind on the ground speed results in the optimal routes (thick lines) to many nodes actually not being the direct connection. This algorithm is repeated until all locations are reached thus providing the quickest route to all location on the grid.

To address whether wind-optimised routes naturally recur with enough spatial regularity such that natural selection could act upon them, we investigated the previously calculated spatially fixed wind-optimised routes across 21 years of historic wind data. Long-term wind data sets suggest that there is consistency in prevailing wind conditions (e.g. Watson et al. 2015). We consider all 21 annual optimal routes between two given locations, of the same calendar month, as a sample of possible routes between these locations for the specific month. We then calculated the travel times, along all 21 routes, and compared them to the travel time along shortest route over the 21 years. This resulted in a series of travelling times that depend on the wind conditions encountered over the 21 years. Assuming a sigmoidal relation between travel time and survival, we determined which of these 21 routes could be favoured by natural selection over these 21 years (Hanski et al. 2000).

\section{MATERIAL AND METHODS}

Our model calculates the optimal route between any two locations in the world travelling over a grid consisting of 65612 cells or nodes. We use a hexagonal grid with the 8 th 
resolution and aperture 3 that consists of hexagons except for 12 pentagons (Sahr et al. 2003). This has the advantage that all points are roughly equally spaced and the nodes are uniformly distributed over the globe. Travel is allowed along straight connections to the six surrounding cells and the second-order neighbours creating 12 travel directions about $30^{\circ}$ apart. The distance to the direct neighbours was on average $95.4 \mathrm{~km}$, whereas the distance to the second-order neighbours was $177.9 \mathrm{~km}$ on average. Wind data were provided by the European Centre for Midrange Weather Forecast (ECMWF; http://www.ecmwf.int/products/data/archive/descriptions/ei/ index.html). The ECMWF ERA-interim atmospheric reanalysis model has a global resolution of 1.5 degrees which corresponds to $167 \mathrm{~km}$ at the equator and provides wind conditions since 1979 until present (Dee et al. 2011). For the interpolation of wind we used four layers of the atmospheric models, $10 \mathrm{~m}$ above ground, 925, 850 and 700 millibar, which correspond to a median altitude of 779,1502 and $3130 \mathrm{~m}$ a.s.1, calculated from a random distributed sample of 10000 locations and start dates. These altitudes cover the range where most migration is observed in radar studies (Kemp et al. 2013). The same wind data have been used in other studies on the effect of wind on the ground speed of birds (Safi et al. 2013). The ground speed $\left(v_{g}\right)$ was calculated as a function of cross wind $\left(w_{c}\right)$ wind support $\left(w_{s}\right)$ and air speed $\left(v_{a}\right)$ as follows:

$v_{g}=\left\{\begin{array}{cl}0 & \text { if } w_{c}>v_{a} \\ 0 & \text { if } w_{c}<v_{a} \& \sqrt{ } v_{a}^{2}-w_{c}^{2}+w_{s}<0 \\ \sqrt{ } v_{a}^{2}-w_{c}^{2}+w_{s} & \text { if } w_{c}<v_{a} \& \sqrt{ } v_{a}^{2}-w_{c}^{2}+w_{s}>0 .\end{array}\right.$

The model begins from a starting location and initiates travel to all surrounding nodes. Once a model node has been reached, travel to the surrounding nodes that have not been reached is initiated. This process continues and reiterates until the destination is reached. By using this speed function and implementing travel over a grid the solution is similar to the 'Dijkstra algorithm' to efficiently calculate the quickest route (Dijkstra 1959, Fig. 1). This algorithm finds the shortest route in a graph, in our case formed by our hexagonal grid. The distances in the graph are determined by travel time calculated using the described speed function. The model was implemented in Java (1.7) and is available on request, analysis were conducted using R (3.0.2).

As a comparison, we calculated travel with similar flight behaviour along a great circle route independent of the used grid defined by 100 intermediate points. Only in a very small proportion (0.0019) of cases we found that the quickest route was the great circle route. This can be a consequence of the grid used, which limits the number of travel directions and number of changes in direction and therefore the approximation of the optimum.

For the sake of computational efficiency, we calculated the model between a limited set of locations in the northern hemisphere and near the equator. These locations we selected to be on land and at least $485 \mathrm{~km}$ apart, by iteratively sampling a location on the grid and then checking whether it was far enough apart from all other locations already selected. We connected 102 locations in the North between $40^{\circ}$ and $60^{\circ}$ latitude with 65 locations in the South between $0^{\circ}$ and $20^{\circ}$ latitude resulting in 2062 location pairs, between which we simulated routes. Location pairs were maximally $50^{\circ}$ longitude apart and within the Americas (390 locations pairs) or AfroEurasian (1672 locations pairs) land masses, to maintain a general North South direction. At this stage, we deliberately exclude the modelling of stopover sites or other obligate migration constraints but acknowledge the fact that such modifications could be included in future models at the cost of the model predictions becoming less generic.

\section{ANALYSIS}

We calculated the optimal routes starting at 21:00 local time on the first of every month from 1990 to 2010 from our northern set of locations to locations in the South in fall (August, September and October) and the reverse in spring (March, April and May). We first investigated whether these routes were substantially more efficient than flying along the shortest spatial connection, the great circle route. To calculate efficiency we used the ratio of the duration of travel along the optimal route to the duration of travel along the great circle route taking the wind conditions in both cases into account.

We fitted a linear model to investigate if there are seasonal or directional patterns of when or where to achieve considerable optimisations. We used altitudinal layer, month, distance, continent, East West direction as predictors. The ratio between travel time over the shortest route and travel time over the wind optimised route was taken as a response variable.

We were interested in routes that were consistently and predictably fast. This was evaluated by calculating travel time throughout the study period at the same starting date over the optimal routes, and was assumed to be maximally 100 days. We postulate that individual survival over the different routes is the driving force for selection of the routes. Although exact measurements on survival during migration are lacking, migration has been shown to be a selective force both through direct survival and carry over effects in specific examples (Newton 2006; Klaassen et al. 2014; Sergio et al. 2014). We therefore assumed travel time $(t)$ to have a sigmoidal relation with mortality, similar to the relation between connectivity and survival in meta-population models (Hanski et al. 2000) and calculated an average survival per year for each route using the travel time over 21 years:

$\Phi=1-\frac{t^{2}}{\lambda+t^{2}}$.

where $\lambda$, the migration mortality is set to the mean travel time across all years squared for every location pair and starting date separately. This means, on an average migration between these two locations half of the individuals would die. The geometric mean of $\Phi$ represents thus the average survival of a route over the period of 21 years as the $n$th root of the product of all survival probabilities (where $n$ is the number of years survival has been measured).

\section{RESULTS}

Our criteria for location pairs resulted in 2062 combinations of departure locations and destinations and a total of 1039248 
routes across all years, months and directions. The optimal routes for each month of the year were spatially very diverse depending on the atmospheric conditions. Travel along the spatially shortest route, following the great circle, was substantially slower than travel along the optimal route calculated by the model. The median ratio of travel time for the optimal routes vs. the shortest routes for all start-destination pairs was 0.73 (25 and $75 \%$ quantile; 0.540 .87 ). The mean realised ground speed along the great circle route was $7.0 \mathrm{~m} \mathrm{~s}^{1} \mathrm{vs}$. $11.9 \mathrm{~m} \mathrm{~s}^{1}$ along the optimal route. The fact that ground speed along the shortest route was considerably lower than the assumed $10 \mathrm{~m} \mathrm{~s}^{1}$ is a consequence of compensating for cross winds. The median increase in length of the optimal route was $13.8 \%$ compared to the shortest route. We tested whether any seasonal or directional patterns could explain travel time differences using a linear model with continent, distance, month, East/West direction and altitude as predictors. The model showed that a considerable proportion of the variance in the travel time ratio between the shortest vs. fastest route can be explained $\left(R^{2}=0.193\right)$. We calculated the variable importance by partitioning the $R^{2}$ values (Grömping 2006), which showed that $78.9 \%$ of the explained variance was explained by the height level used. Relatively larger travel time optimisations can be reached at higher altitudes (Table 1).

Most $(84.8 \%)$ location pairs had at least one route with a higher average survival than the great circle (e.g. Fig. 2) over the 21 years. The median ratio between survival rates following the best route vs. the great circle route was 1.083 indicating an advantage of choosing a spatially fixed route due to the advantages provided by the wind conditions encountered en route over a period of 21 years, based upon empirical wind data. Despite the median length increase for the wind-optimised routes with the highest survival values by $10.4 \%$, the median time gain along these routes was still 0.78 days (or $91.1 \%$ of the travel time along the shortest route). The average ground speed over the route with the maximal survival was $8.8 \mathrm{~m} \mathrm{~s}^{1}$, compared to $7.0 \mathrm{~m} \mathrm{~s}^{1}$ on the shortest route. These increases hold across height levels although the effect size decreases with increasing altitude (Table 1).

The routes with maximum survival differed considerably in the spatial location between travel directions/season (Fig. 3). There is a higher correlation between height levels in the spatial position of the routes, especially between the 10 meter above ground level, 925 and 850 millibar level. The various routes with highest survival connecting different start and end locations, over the period of 21 years of wind data, converged (a)
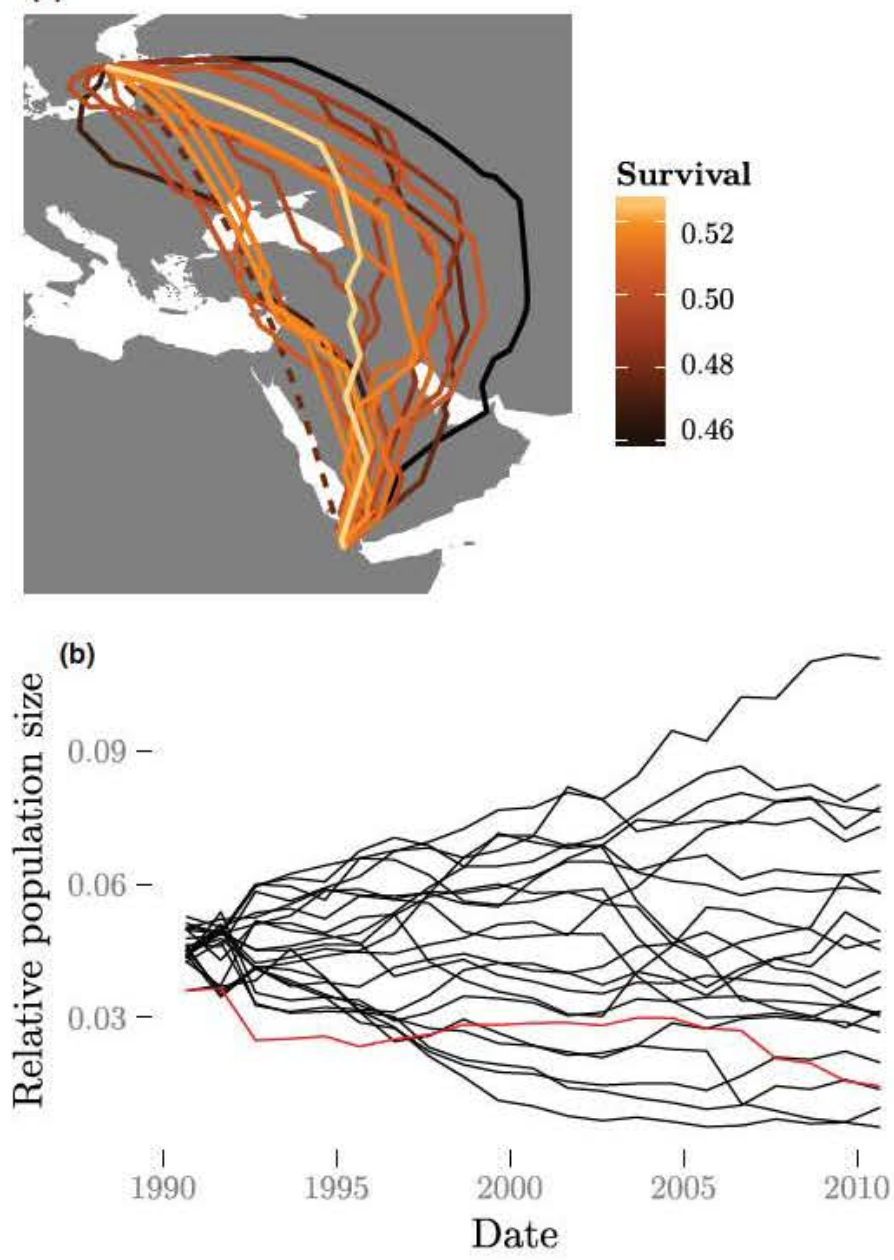

Figure 2 All optimal routes starting on the first of September connecting Sweden $\left(18.3^{\circ}, 59.3^{\circ}\right)$ to Ethiopia $\left(14.6^{\circ}, 12.0^{\circ}\right)$. The great circle route corresponds to the red/dashed line. (a) shows all tracks that have been optimal in 1 year coloured according to their average survival over all 21 years. (b) shows the proportion of the population following specific routes over time, each spatially fixed route is represented by a line. Many routes consistently outperformed the great circle route. The high survival routes all followed a similar trajectory running east of the shortest route.

spatially into flyways. Finally, for many pairs of locations, the optimal routes for journeys from North to South were located more easterly compared to the routes in the opposite direction connecting the same locations, resulting in loop migrations.

Table 1 An overview of the statistics per altitudinal layer and the overall averages. The following parameters are used $t$ is travel time, $d$ distance, $v$ speed and $\Phi$ survival. Subscripts refer to the route type; opt stands for the optimal route, gc for the great circle route and max for the route with maximal survival

\begin{tabular}{|c|c|c|c|c|c|c|c|c|c|c|c|c|c|}
\hline & \multicolumn{6}{|c|}{ Individual tracks } & \multicolumn{7}{|c|}{ Route summary over years } \\
\hline & $\frac{t_{\text {apti }}}{t_{g e}}\left(Q_{1}\right.$ & $\left.Q_{3}\right)$ & $\overline{v_{g c}}\left(\mathrm{~m} \mathrm{~s}^{-1}\right)$ & $v_{o p t}^{-}\left(\mathrm{m} \mathrm{s}^{-1}\right)$ & $\frac{d_{\text {gpt }}}{d_{g c}}$ & $P\left(\Phi_{\max }>\Phi_{g c}\right)$ & $\frac{\Phi_{\max }}{\Phi_{g c}}$ & $\frac{d_{\max }}{d_{3 x}}$ & $\overline{t_{g c}}$ & $t_{\max }^{-}($days $)$ & $\frac{t_{\max }}{t_{g s}}$ & $v_{g c}\left(\mathrm{~m} \mathrm{~s}^{-1}\right)$ & $v_{\max }^{-}\left(\mathrm{m} \mathrm{s}^{-1}\right)$ \\
\hline $10 \mathrm{~m}$ a.g.l. & $0.87(0.77$ & $70.93)$ & 8.50 & 11.27 & 1.09 & 0.80 & 1.03 & 1.06 & 0.24 & & 0.96 & 8.50 & 9.76 \\
\hline 925 millibar & $0.67(0.48$ & $80.83)$ & 6.48 & 11.92 & 1.16 & 0.92 & 1.14 & 1.13 & 1.39 & & 0.86 & 6.48 & 8.87 \\
\hline 850 millibar & $0.69(0.52$ & $20.83)$ & 6.64 & 11.96 & 1.16 & 0.88 & 1.10 & 1.12 & 1.04 & & 0.89 & 6.64 & 8.52 \\
\hline 700 millibar & $0.64(0.42$ & $20.81)$ & 6.32 & 12.31 & 1.18 & 0.79 & 1.10 & 1.13 & 1.24 & & 0.89 & 6.32 & 7.87 \\
\hline Overall & $0.73(0.54$ & 40.87 ) & 6.98 & 11.86 & 1.14 & 0.85 & 1.08 & 1.10 & 0.78 & & 0.91 & 6.98 & 8.75 \\
\hline
\end{tabular}




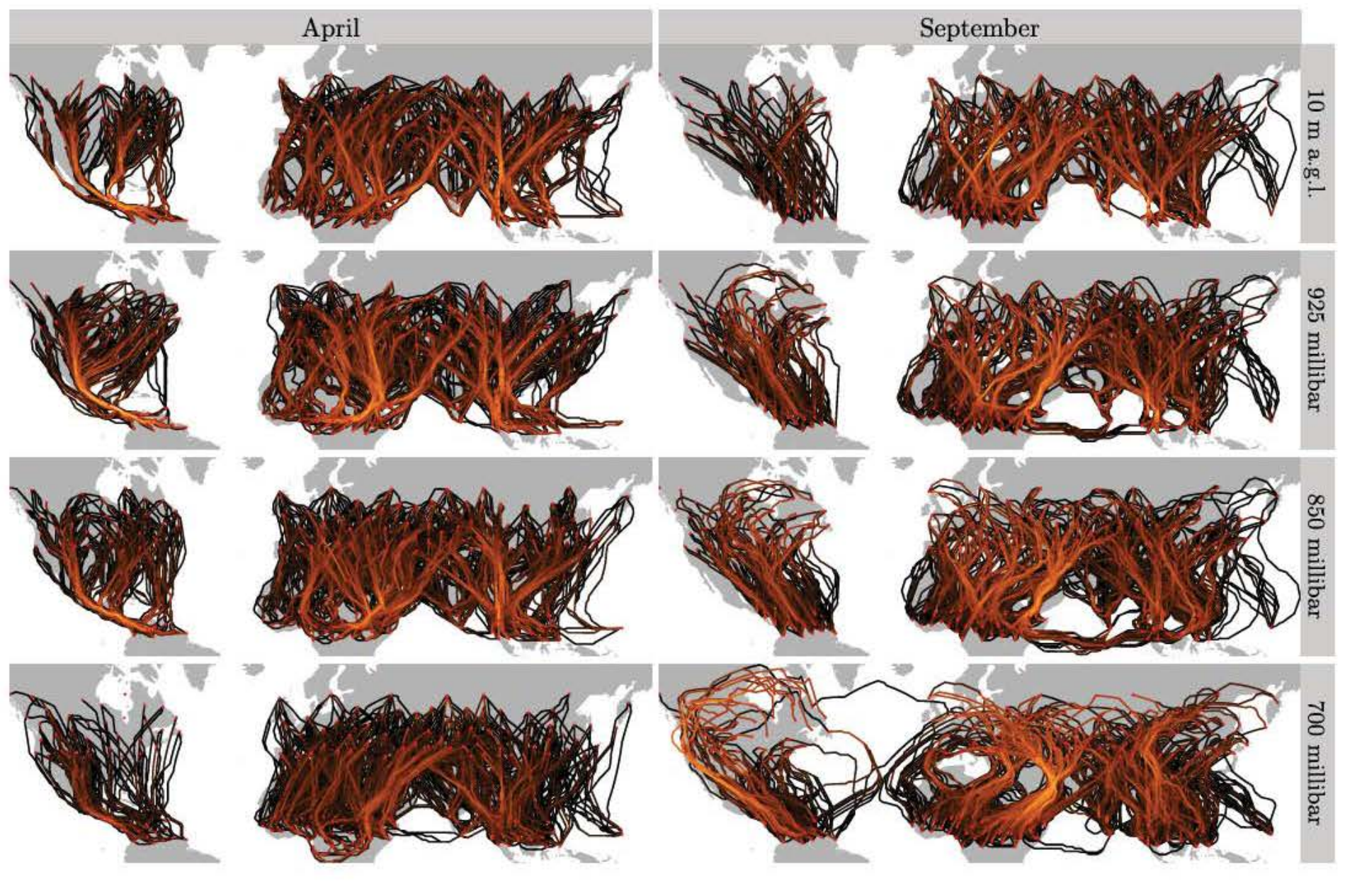

$$
\text { Number of routes }-1-10-50-100-200
$$

Figure 3 The routes with the highest survival, excluding those that follow the great circle route, between locations going from North to South in September and from South to North in April separated in altitudinal layers. There are clear differences between seasons, where in September many routes between the Americas are far out on the Atlantic Ocean, whereas in April the optimal routes are closer to the continent. The routes between Africa and Europe are more eastwards in September than in April. The start and end locations are highlighted in red. The number of routes through each segment are indicated by colour.

\section{DISCUSSION}

Taking wind conditions into account, we found that a route other than the spatially shortest route along the great circle almost always allowed for faster migration, often with a substantial speed increase. Even when looking over longer time periods, spatially fixed routes provided a considerable advantage over the great circle routes. In autumn we found that most routes from Europe to Africa are predominantly easterly located which corresponds to earlier findings that show the eastern route to have a higher survival in simulations, except for when refuelling and altitudinal optimisation were possible (Erni et al. 2005). We also find that the potential for optimisation is strongly dependent on the flight altitude, where higher altitudes allow for larger optimisations. The differences between altitudinal layers could be both due to differences in wind speed and/or regularity. Although each individual bird likely lacks the information necessary to find the optimal route for a specific year, the spatio-temporal predictability evi- dently present in weather systems means that routes could be subject to natural selection. Route use could then be inherited genetically or spread through populations via cultural inheritance.

Several patterns in Fig. 3 strikingly match observed migrations. We predicted, for example southward migrations over the Atlantic ocean directly towards the coast of South America. These routes seem to correspond to the recently documented migration routes of the blackpoll warbler (Setophaga striata, DeLuca et al. 2015). Similarly the Hudsonian godwit (Limosa haemastica) migrates across the Atlantic to its stopover sites in South America (Senner 2013). These similarities are especially interesting because all these species make long continuous flights, very similar to the assumption underlying our model. In the migratory routes within Europe to Africa we find that the southbound migrations are more easterly compared to the northbound migrations. This general pattern is found, for example in the common cuckoo, which shows a clockwise loop migration (Willemoes et al. 2014). Similarly, in 
North America, southbound migrations are more eastern than the northbound migrations, a pattern also observed in both individual trajectories around the Gulf of Mexico (Bradley et al. 2014) as well as average migrations (La Sorte et al. 2013). In Asia, we find prominent migration routes through the Philippines and Malaysian peninsula much like the existing flyways (Newton 2007). Even though these examples should not be interpreted as evidence that our hypothesised mechanisms underlying these routes are correct, they are nevertheless consistent with the hypothesis that the optimisation on wind could play an important role in bird migration and defining migratory flyways. Other well-known migration corridors, such as the one crossing over the strait of Gibraltar (Newton 2007), might not appear as dominant as expected. This is not unexpected as we anticipated the absence of some known routes due to the omission of various important factors, such as stopover site availability or avoiding to cross open ocean areas such as the Mediterranean. These factors surely influence migration routes in certain areas of the world. In many cases, the optimal route will be dependent on various factors that balance both wind borne advantages and stopover possibilities. Routes that are not reflected in the model might also depend on these other factors which does not imply that wind is unimportant. Despite its simplicity, our model emphasises two pivotal aspects of global bird migrations: first, the central importance of optimising the atmospheric conditions over minimising distance. Second, the model illustrates the fact that wind conditions, despite their inherently dynamic nature, appear to be sufficiently predictable to be subject to natural selection or cultural transmission.

The model we propose provides fundamental insight into some poorly understood general patterns and regularities of animal aerial migrations and movements. All journeys, both along the optimal and the spatially shortest routes, were modelled according to the same assumptions about the flight behaviour of birds. Therefore, we are confident that the general qualitative conclusions drawn from our analysis are robust, even more so as we know that several adaptive behavioural responses to cope with changing environmental conditions were not considered in our model. For example we falsely, but out of simplicity, assumed that birds interrupt flight whenever they encounter stronger head or cross winds than their flight speed allowed them to move with. The model assumes a simple additive relationship between air speed, wind speed and ground speed where the modelled birds would not adjust their behaviour. However, birds should, and do, display a more complex reaction towards wind speed and direction, for example in case of stronger head winds they increase air speed (Pennycuick 1978; Shamoun-Baranes et al. 2007; Safi et al. 2013). However, such optimisation of ground speed will require additional effort, but should produce qualitatively similar results in our model.

It is well possible that following a spatially fixed route provides an even larger advantage when departure time is adjusted in relation to specific weather conditions. This has, for example been shown for migrations over the Atlantic sea to South America by the blackpoll warblers (DeLuca et al. 2015) or for bar-tailed godwits travelling to New Zealand (Conklin \& Battley 2011). Our simulations, however, did not include any behavioural adjustments, which could even further increase the performance of specific spatially fixed routes. For example a drop in temperature, air pressure or an increase in wind speed in a specific direction could indicate a favourable weather front and thus provide the birds with some forecasting possibilities (Richardson 1990). Such additional adjustments could be modelled by comparing the optimal route for a range of departure dates or making departure dependent on local environmental conditions. We think that the additional optimisations of air speed and departure time are likely to even further augment the relative difference between the shortest route and the route with maximal survival. Optimising departure, for example will allow birds to migrate with support of specific weather systems, while favourable winds might not occur along the shortest route. Besides optimising the timing of departure, birds could further optimise their flight altitude (Liechti \& Bruderer 1998; Dokter et al. 2013). Our model could be extended to optimise altitude, but this would be met with a considerably increased computational effort. At the same time, optimising altitude would allow for larger decreases in travel time if stronger and more predictable winds are available at higher altitude. Generally, higher altitudes have stronger winds but their predictability is less clear (Liechti 2006; Shamoun-Baranes et al. 2010). Furthermore, some birds will increase air speed at higher altitudes due to lower air pressure (Schmaljohann \& Liechti 2009). This will mean that the difference between lower and higher altitudes we found is possibly even more pronounced.

An alternative optimisation would include navigational decisions that depend on current local weather conditions and in that way optimise the route with respect to global weather conditions (McLaren et al. 2014). Conditional route choice could help find the optimal route when temporal patterns in the weather occur. Such a strategy does not exclude the evolution of fixed routes. Rather, conditional choices could be between various fixed migration routes. The possibility to optimise using conditional route choice would probably be best investigated in a different model where such choices are explicitly incorporated.

To our knowledge, this study is the first to investigate the presence of globally predictable migration routes with respect to wind conditions that birds could follow. In contrast to earlier studies on the influence of wind on long distance travel, we did not limit ourselves to the great circle route between locations (Shamoun-Baranes et al. 2010) or to fixed headings (Erni et al. 2005; Vrugt et al. 2007) while optimising the route in respect to the wind conditions. Finding the routes based on our model requires more advanced navigational abilities in contrast to these earlier studies. These routes could be followed either using map navigation, where the birds know in absolute position at any given time where on the globe they are and where they want to go to. Birds could also navigate on the basis of compass navigation, where they would constantly compensate for the wind during the orientation towards a specific goal and apply a kind of wind corrected compass navigation. Particularly for small passerines, laboratory experiments and studies of hybrids suggest that complex migratory routes, including changes in heading and travel 
direction, can be endogenous and innate (Helbig 1996; Delmore \& Irwin 2014).

Earlier studies that have included stopovers as a part of the simulations (Erni et al. 2005; Vrugt et al. 2007) have used the simulations to predict stopover usage (Shamoun-Baranes et al. 2010) or even optimised multiple objectives (Vrugt et al. 2007). In this study, we did not include stopovers, and in case of uniformly distributed refuelling possibilities we do not expect large influences on the spatial distribution of the route. For species with specific stopover ecologies, however, such as waders preferring coastal areas, or in case of generally adverse habitat such as deserts this might restrict the possible routes.

The model we present was optimised for birds that fly using a predominately flapping flight mode. The modelling framework could, however, be adjusted to accommodate specificity of other types of flight by including more complex and thus more realistic functions better representing a specific species' flight ecology. It is even possible to extend it to birds with entirely different flight strategies, as long as the parameters determining ground speed as a function of atmospheric conditions are well described (e.g. Bohrer et al. 2012). Finally, the model is not restricted to atmospheric information and could be applied to marine animals travelling in and with the ocean currents.

Our model predicts that the atmospheric conditions play a large role in determining the efficiency of migratory routes. Across 21 years of wind data, travel routes were stable enough to allow evolutionary processes to act at population levels. With this model it is possible to compare realised trajectories of birds travelling between known locations at specific times in the year and to obtain an objective measure of migratory efficiency. Finally, this model provides a base for disentangling the effects of travel efficiency from factors other than wind support, potentially highlighting the importance of the availability of suitable stopover sites or other vital resources along long journeys.

\section{ACKNOWLEDGEMENTS}

We thank Rechenzentrum Garching of the Max Planck Society for supporting the computer cluster used. The International Max Planck Research School for Organismal Biology supported B.K. We furthermore thank three reviewers and the editor for their valuable comments and Teague O'Mara for proof reading the manuscript.

\section{AUTHOR CONTRIBUTIONS}

All authors conceived the study. BK and RW performed the study; BK wrote the initial draft to which all authors contributed.

\section{REFERENCES}

Alerstam, T. (1979). Wind as selective agent in bird migration. Ornis Scand., 10, 7693.

Alerstam, T. (2011). Optimal bird migration revisited. J. Ornithol., 152, 523.

Alerstam, T., Hedenstrom, A. \& Åkesson, S. (2003). Long distance migration: evolution and determinants. Oikos, 103, 247260
Berthold, P. (2001). Bird Migration: A General Survey. Oxford University Press, Oxford.

Biebach, H., Biebach, I., Friedrich, W., Heine, G., Partecke, J. \& Schmidl, D. (2000). Strategies of passerine migration across the Mediterranean Sea and the Sahara Desert: a radar study. Ibis, 142, 623634.

Bohrer, G., Brandes, D., Mandel, J.T., Bildstein, K.L., Miller, T.A., Lanzone, M. et al. (2012). Estimating updraft velocity components over large spatial scales: contrasting migration strategies of golden eagles and turkey vultures. Ecol. Lett., 15, 96103.

Bradley, D.W., Clark, R.G., Dunn, P.O., Laughlin, A.J., Taylor, C.M., Vleck, C. et al. (2014). Trans Gulf of Mexico loop migration of tree swallows revealed by solar geolocation. Curr. Zool., 60, 653659.

Conklin, J.R. \& Battley, P.F. (2011). Impacts of wind on individual migration schedules of New Zealand bar tailed godwits. Behav. Ecol., 22, 854861 .

Dee, D.P., Uppala, S.M., Simmons, A.J., Berrisford, P., Poli, P., Kobayashi, S. et al. (2011). The ERA Interim reanalysis: configuration and performance of the data assimilation system. Q.J. Roy. Meteor. Soc., 137, 553597.

Delmore, K.E. \& Irwin, D.E. (2014). Hybrid songbirds employ intermediate routes in a migratory divide. Ecol. Lett., 17, 12111218.

DeLuca, W.V., Woodworth, B.K., Rimmer, C.C., Marra, P.P., Taylor, P.D., McFarland, K.P. et al. (2015). Transoceanic migration by a $12 \mathrm{~g}$ songbird. Biol. Lett., 11, 20141045.

Dijkstra, E.W. (1959). A note on two problems in connexion with graphs. Numer. Math., 1, 269271.

Dokter, A.M., Shamoun Baranes, J., Kemp, M.U., Tijm, S. \& Holleman, I. (2013). High altitude bird migration at temperate latitudes: a synoptic perspective on wind assistance. PLoS ONE, 8, e52300.

Egevang, C., Stenhouse, I.J., Phillips, R.A., Petersen, A., Fox, J.W. \& Silk, J.R.D. (2010). Tracking of Arctic terns Sterna paradisaea reveals longest animal migration. Proc. Natl Acad. Sci., 107, 20782081.

Erni, B., Liechti, F. \& Bruderer, B. (2005). The role of wind in passerine autumn migration between Europe and Africa. Behav. Ecol., 16, 732740 .

Gehrold, A., Bauer, H.G., Fiedler, W. \& Wikelski, M. (2014). Great flexibility in autumn movement patterns of European gadwalls Anas strepera. J. Avian Biol., 45, 131139.

Gill, R.E., Tibbitts, T.L., Douglas, D.C., Handel, C.M., Mulcahy, D.M., Gottschalck, J.C. et al. (2009). Extreme endurance flights by landbirds crossing the Pacific Ocean: ecological corridor rather than barrier? Proc. Biol. Sci., 276, 447457.

Gromping, U. (2006). Relative importance for linear regression in R: the package relaimpo. J. Stat. Softw., 17, 127.

Hanski, I., Alho, J. \& Moilanen, A. (2000). Estimating the parameters of survival and migration of individuals in metapopulations. Ecology, 81, 239251.

Hawkes, L.A., Balachandran, S., Batbayar, N., Butler, P.J., Frappell, P.B., Milsom, W.K. et al. (2011). The trans Himalayan flights of bar headed geese (Anser indicus). Proc. Natl Acad. Sci., 108, 95169519.

Hedenstrom, A. (2008). Adaptations to migration in birds: behavioural strategies, morphology and scaling effects. Philos. Trans. R. Soc. Lond. B Biol. Sci., 363, 287299.

Helbig, A.J. (1996). Genetic basis, mode of inheritance and evolutionary changes of migratory directions in palaearctic warblers (Aves: Sylviidae). J. Exp. Biol., 199, 4955.

Kemp, M.U., Shamoun Baranes, J., Dokter, A.M., van Loon, E. \& Bouten, W. (2013). The influence of weather on the flight altitude of nocturnal migrants in mid latitudes. Ibis, 155, 734749.

Klaassen, R.H.G., Alerstam, T., Carlsson, P., Fox, J.W. \& Lindstrom, A. (2011). Great flights by great snipes: long and fast non stop migration over benign habitats. Biol. Lett., 7, 833835.

Klaassen, R.H.G., Hake, M., Strandberg, R., Koks, B.J., Trierweiler, C., Exo, K.M. et al. (2014). When and where does mortality occur in migratory birds? Direct evidence from long term satellite tracking of raptors. J. Anim. Ecol., 83, 176184. 
La Sorte, F.A., Fink, D., Hochachka, W.M., DeLong, J.P. \& Kelling, S. (2013). Population level scaling of avian migration speed with body size and migration distance for powered fliers. Ecology, 94, 18391847.

Liechti, F. (2006). Birds: blowin' by the wind? J. Ornithol., 147, 202211.

Liechti, F. \& Bruderer, B. (1998). The relevance of wind for optimal migration theory. J. Avian Biol., 29, 561568.

Liechti, F., Hedenstrom, A. \& Alerstam, T. (1994). Effects of sidewinds on optimal flight speed of birds. J. Theor. Biol., 170, 219225.

Liedvogel, M., Ảkesson, S. \& Bensch, S. (2011). The genetics of migration on the move. Trends Ecol. Evol., 26, 561569.

Lindstrom, A., Gill, R.E., Jamieson, S.E., McCaffery, B., Wennerberg, L., Wikelski, M. \& Klaassen, M. (2011). Are fueling conditions in Alaska driving the movement of juvenile sharp tailed sandpipers? Condor, 113, 129139.

McLaren, J.D., Shamoun Baranes, J., Dokter, A.M., Klaassen, R.H.G. \& Bouten, W. (2014). Optimal orientation in flows: providing a benchmark for animal movement strategies. J. R. Soc. Interface, 11, 20140588.

Mueller, T., O'Hara, R.B., Converse, S.J., Urbanek, R.P. \& Fagan, W.F. (2013). Social learning of migratory performance. Science, 341, 999 1002 .

Newton, I. (2006). Can conditions experienced during migration limit the population levels of birds? J. Ornithol., 147, 146166

Newton, I. (2007). The Migration Ecology of Birds. Academic Press, London.

Pennycuick, C.J. (1978). Fifteen testable predictions about bird flight. Oikos, 30, 165176.

Pulido, F., Berthold, P., Mohr, G. \& Querner, U. (2001). Heritability of the timing of autumn migration in a natural bird population. Proc. $R$. Soc. Lond. B Biol. Sci., 268, 953959.

Richardson, W.J. (1990). Timing of bird migration in relation to weather: updated review. In: Bird Migration (ed. Gwinner, E.). Springer, Berlin, Heidelberg, pp. 78101 .

Safi, K., Kranstauber, B., Weinzierl, R., Griffin, L., Rees, E.C., Cabot, D. et al. (2013). Flying with the wind: scale dependency of speed and direction measurements in modelling wind support in avian flight. Mov. Ecol., 1, 4 .

Sahr, K., White, D. \& Kimerling, A. (2003). Geodesic discrete global grid systems. Cartogr. Geogr. Inf. Sci., 30, 121134.

Sapir, N., Butler, P.J., Hedenstrom, A. \& Wikelski, M. (2011). Energy gain and use during animal migration. In: Animal Migration (eds Milner Gulland, E., Fryxell, J.M. \& Sinclair, A.R.). Oxford University Press, Oxford, pp. 5267.
Schmaljohann, H. \& Liechti, F. (2009). Adjustments of wingbeat frequency and air speed to air density in free flying migratory birds. $J$. Exp. Biol., 212, 36333642.

Senner, N. (2013). The effects of global climate change on long distance migratory birds. PhD Thesis, Cornell University.

Sergio, F., Tanferna, A., Stephanis, R.D., Jiménez, L.L., Blas, J., Tavecchia, G. et al. (2014). Individual improvements and selective mortality shape lifelong migratory performance. Nature, 515, 410413.

Shamoun Baranes, J., Van Loon, E., Liechti, F. \& Bouten, W. (2007). Analyzing the effect of wind on flight: pitfalls and solutions. J. Exp. Biol., 210, 8290 .

Shamoun Baranes, J., Leyrer, J., van Loon, E., Bocher, P., Robin, F., Meunier, F. \& Piersma, T. (2010). Stochastic atmospheric assistance and the use of emergency staging sites by migrants. Proc. Biol. Sci, $277,15051511$.

Vrugt, J.A., Van Belle, J. \& Bouten, W. (2007). Pareto front analysis of flight time and energy use in long distance bird migration. J. Avian Biol., 38, 432442.

Watson, S.J., Kritharas, P. \& Hodgson, G.J. (2015). Wind speed variability across the UK between 1957 and 2011. Wind Energy, 18, 21 42.

Wikelski, M., Tarlow, E.M., Raim, A., Diehl, R.H., Larkin, R.P. \& Visser, G.H. (2003). Avian metabolism: costs of migration in free flying songbirds. Nature, 423, 704704.

Willemoes, M., Strandberg, R., Klaassen, R.H.G., Tøttrup, A.P., Vardanis, Y., Howey, P.W. et al. (2014). Narrow front loop migration in a population of the common cuckoo cuculus canorus, as revealed by satellite telemetry. PLOS ONE, 9, e83515. 